This item was submitted to Loughborough's Research Repository by the author.

Items in Figshare are protected by copyright, with all rights reserved, unless otherwise indicated.

\title{
Adaptive subframe allocation for next generation multimedia delivery over hybrid LTE unicast broadcast
}

PLEASE CITE THE PUBLISHED VERSION

http://dx.doi.org/10.1109/TBC.2016.2570020

PUBLISHER

(c) IEEE

VERSION

AM (Accepted Manuscript)

LICENCE

CC BY-NC-ND 4.0

\section{REPOSITORY RECORD}

Christodoulou, Louis, Omar Abdul-Hameed, Ahmet Kondoz, and J. Calic. 2016. "Adaptive Subframe Allocation for Next Generation Multimedia Delivery over Hybrid LTE Unicast Broadcast”. Loughborough University. https://hdl.handle.net/2134/21818. 


\title{
Adaptive Subframe Allocation for Next Generation Multimedia Delivery over Hybrid LTE Unicast Broadcast
}

\author{
Louis Christodoulou, Omar Abdul-Hameed, Ahmet M. Kondoz and Janko Calic
}

\begin{abstract}
The continued global roll-out of Long Term Evolution (LTE) networks is providing mobile users with perpetually increasing ubiquitous access to a rich selection of high quality multimedia. Interactive viewing experiences including 3D or Free-Viewpoint Video (FVV) require the synchronous delivery of multiple video streams. This paper presents a novel Hybrid Unicast Broadcast Synchronisation (HUBS) framework to synchronously deliver multi-stream content. Previous techniques on hybrid LTE implementations include staggered Modulation and Coding Scheme (MCS) grouping, Adaptive Modulation Coding (AMC) or implementing error recover techniques; the work presented here instead focuses on dynamic allocation of resources between unicast and broadcast, improving stream synchronisation as well as overall cell resource usage. Furthermore, the HUBS framework has been developed to work within the limitations imposed by the LTE specification. Performance evaluation of the framework is performed through the simulation of probable future scenarios, where a popular live event is broadcast with stereo 3D or multi-angle companion views interactively offered to capable users. The proposed framework forms a 'HUBS Group' that monitors the radio bearer queues to establish a time lead or lag between broadcast and unicast streams. Since unicast and broadcast share the same radio resources, the number of subframes allocated to the broadcast transmission are then dynamically increased or decreased to minimise the average lead/lag time offset between the streams. Dynamic allocation showed improvements for all services across the cell, whilst keeping streams synchronised despite increased user loading.
\end{abstract}

Index Terms-Multicast, LTE, E-MBMS, H.264 MVC, cellular networks.

\section{INTRODUCTION}

D ATA TSUNAMI; the term often being used to describe the impending wave of mobile data traffic set to overwhelm mobile networks. Mobile data traffic is expected to increase nearly 11-fold between 2013 and 2018, with video data forecast to account for $70 \%$ of this [1]. Industry analysts forecast that by 2018 , network capacity will be exceeded several times daily, resulting in loss of service for users [2]. Larger and higher resolution screens, greater processing power and reduced cost of smart phone and tablet devices are all thought to be contributing factors to the greater content and quality demand from the network [3]. Mobile media consumption has also placed increasing pressure on the spectrum resources assigned to traditional Digital Television (DTV) services [4].

L.Christodoulou and J.Calic are with University of Surrey, Guildford, Surrey, UK (E-Mail: 1.christodoulou@surrey.ac.uk; j.calic@surrey.ac.uk)

O. Abdul-Hameed and A. Kondoz are with Loughborough University, London, UK (E-mail: o.abdul-hameed@lboro.ac.uk; a.kondoz@lboro.ac.uk)
The concept underpinning the work proposed in this paper is formed based on the observation of two diverging trends: Long Term Evolution (LTE) enhanced Multimedia Broadcast Multicast Service (eMBMS) [5] is expected to play a significant role in reducing the burden of delivering next generation multimedia to mobile devices. Due to the way in which radio resources are allocated, there may exist a scenario where eMBMS deployment of unpopular content, severely reduces the radio resource efficiency LTE broadcast can offer. To this end, interest from multiple users, in the same content is required for eMBMS deployment to present efficiency improvements over unicast. Meanwhile, content creators and broadcasters are diverging from traditional single stream offerings, increasingly providing individual users greater choice to personalise the way in which they consume content. These enhanced offerings open up additional revenue streams for mobile operators and content creators who can offer 'premium services' to subscribers, thereby enhancing a live broadcast event. Example applications include, Ultra High Definition Television (UHDTV), 3D Television, Free Viewpoint Television (FVT) and Multi/Companion Screen viewing.

This poses both a unique and complex problem, since even popular live broadcast content may have users divided across several different streams. Furthermore, with myriad mobile device models, each with varying performance and capabilities, further device specific streams may be required and the problem sees further complication. Consequently, with the pool of interested users diluted across multiple streams, the ability to efficiently cater for this increased choice with LTE broadcast alone increasingly diminishes, as multiple broadcast streams will have to be established each with fewer users and thus lower efficiency. Unlike broadcast channels, unicast, or point-to-point (p-t-p) radio bearers are able to rapidly and dynamically conform to fast changing channel conditions, continually maximising spectral efficiency offered. Thus, it is proposed, since the core content for the pool of interested users is fundamentally the same, all users may subscribe to a single base broadcast stream, whilst having the ability to individually fetch unicast support streams which when combined are able to offer the enhanced or tailored service.

The approach presented in this paper explores a multimedia centric, hybrid use of unicast and broadcast delivery methods. While previous work on LTE based hybrid implementations attempt to maximise cell Spectral Efficiency (SE) through techniques such as staggered Modulation and Coding Scheme (MCS) grouping, broadcast implementations of Adaptive Mod- 


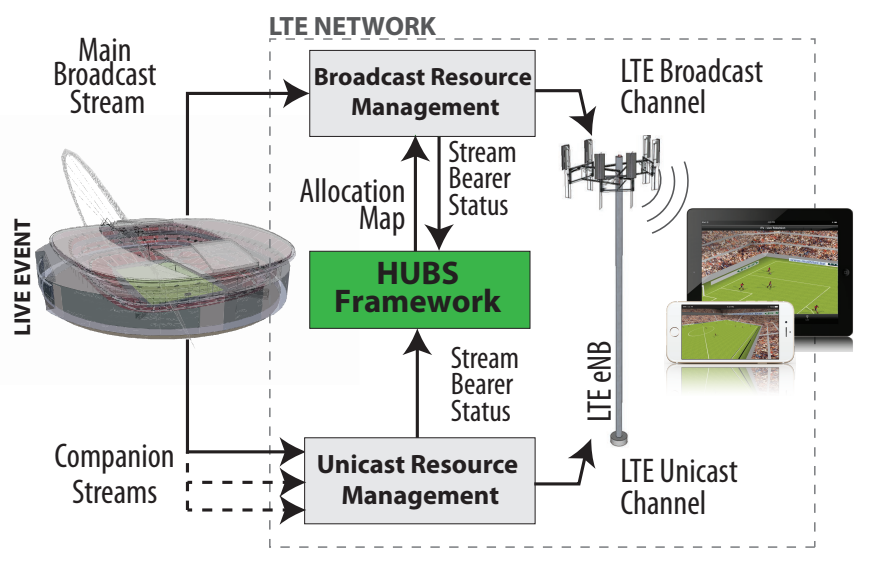

Fig. 1. Proposed HUBS framework with reference scenario

ulation Coding (AMC) or implementing error recovery techniques, the work here instead focuses on the balancing of resources between unicast and broadcast services. By monitoring the status of the users' bearer queues within a Hybrid Unicast Broadcast Synchronisation (HUBS) group, resources are dynamically allocated between unicast and broadcast services, providing stream synchronisation, maintaining Quality of Service (QoS) parameters and optimising the cell based on current user position and load conditions. This is achieved through enhancements to the resource allocation mechanisms already established within the technical design of the LTE standard, further explained in Section III. Also unique to the research is the level to which it has been implemented to respect the limitations presented by the LTS eMBMS design specification, particularly pertaining to the frequency at which updates may be made to the broadcast configuration. Essentially, the research presented could theoretically be deployed by a mobile network operator as a software update within an eMBMS capable LTE network.

Despite the increasing quantity of research as well as consumer popularity of multi-stream multimedia, such as 3D Television, Free Viewpoint Television (FVT) [6] and Multi/Companion Screen viewing, to date, no study has been conducted into the joint delivery of this content using a hybrid unicast and broadcast LTE services.

Based on the trends presented above, two future typical realworld scenarios are simulated, where a popular live broadcast is enhanced with additional viewing options. The first offers the main broadcast coverage of a football match via LTE broadcast, with users able to receive and interactively switch between available companion views delivered by unicast transmission. A high level overview example of this scenario is shown in Figure 1. The second scenario explores stereoscopic coverage of a popular live event. The left view of the 3D stream is broadcast using eMBMS in high definition within a cell experiencing high user demand. A selection of users in the cell with $3 \mathrm{D}$ enabled devices are then catered for via unicast with the right view (enhancement stream), providing them with a stereoscopic 3D experience.

The remainder of this paper is presented as follows. Section
II provides a background and summery of Related Work. Section III covers details on eMBMS service implementation, with focus on how resources are allocated between unicast and broadcast services within the LTE standard. This forms a foundation for the description of the developed framework as well as simulation elements that are detailed in Section III. Section IV provides details of the two simulation scenarios including an analysis of the compiled video sequences. Section $\mathrm{V}$ outlines the simulation details and provides a performance evaluation of the simulation results. Finally, section VI forms the concluding arguments and suggestions for future enhancements.

\section{BACKGROUND AND RELATED WORK}

Generally speaking, much of the research effort on LTE broadcast has been centered around Spectral Efficiency (SE). Reduced SE of eMBMS versus unicast transmission is primarily caused by a property all broadcast systems suffer from: Performance limits are based on the User Equipment (UE) with the weakest radio channel quality [7]. The channel quality dictates which Modulation and Coding Scheme (MCS) can be used without resulting in excessively erroneous reception. A weaker channel will call for a more robust MCS selection, with greater coding and lower order modulation. This will come at the cost of reduced data transmission and therefore reduced SE. In LTE, by default, once an MCS is chosen it remains for the duration of the eMBMS session. It is therefore chosen independently of the current distribution of users within the cell, instead, the consideration that any new users may appear within the cell during the broadcast is made. Therefore any MCS chosen will ensure robust coverage across the entirety of the cell, including more interference prone cell edges. To mitigate the loss this causes, work in [7] proposed the use of fast Adaptive Modulation Coding (AMC) based on feedback from UEs for a more informed selection of MCS. The authors also explored the use of Hybrid Automatic Repeat Request (HARQ) feedback for error recovery. Simulations showed gains in UE throughput of up to $25 \%$ and $70 \%$ for fast AMC and HARQ respectively. More recently, an adaptive resource allocation policy for multicast is proposed in [8], where Araniti et al. group users requesting multicast services into subgroups by utilising an optimisation problem approach. Following this, AMC is applied accordingly on a subgroup basis, successfully recovering some of the multi-user diversity gains lost through Conventional Multicast Schemes (CMS).

The other key variable that can influence channel quality is transmission power. In [9], the authors use unicast Channel Quality Indicator (CQI) feedback mechanism to determine power saving adjustments for a group of UEs subscribed to the same eMBMS content. Although these are significant gains, the benefits presented by systems with group channel quality feedback tend to diminish at an increasing rate as user numbers increase, an effect that can be clearly seen in [7].

Given the forecast data trends, research in the delivery of future broadcast television over cellular networks has recently gained traction. Walker et al. in [4] identifies that "traffic growth is far exceeding the growth in available bandwidth". 
Furthermore, rather than directly targeting bandwidth from DTV, the paper presents intuitive methods to share bandwidth, thus providing a greater aggregate efficiency between the two services. Perhaps most interestingly, analysis in [4] shows LTE broadcast services fair considerably better in dense urban environments than less dense suburban. Furthermore, it is shown that for two users of common content in a suburban deployment, unicast services at $2 \mathrm{GHz}$ will deliver the content with greater SE, whereas, at UHF $(700 \mathrm{MHz})$ and $850 \mathrm{MHz}$ frequencies, broadcast is more spectrally efficient for the same environment. This is put down to the improved propagation characteristics of the signals at lower frequencies when presented with the larger Inter-Site Distances (ISDs) of cells in a suburban cell deployment. In [10], Crussire et al. describe the results of previous attempts at proposing "pure broadcast solutions to the mobile ecosystem" as being "vain". The paper continues to present some lessons learnt from these previous attempts: non-3GPP chipsets are unlikely to be embedded in devices without having mobile vendors in the loop from day one, cost issues prevent deployment and management of a second network and new services should be offered to properly assess interest in a new broadcast solution. The paper concludes presenting a common physical layer based on EMBMS and DVB-T2 standards [11].

An interesting case study on DTV distribution over cellular networks is carried out in [12]. Here Shi et al. carried out analysis on a hypothetical "CellTV" network, operating in the 470$790 \mathrm{MHz}$ spectrum to provide services to rural and urban areas in Sweden in the year 2020. The study accounted for both viewing patterns and expected advances in technology. The "CellTV" network could make use of both unicast and broadcast capabilities, and, for broadcast employing an approach using layered Multicast Broadcast Single Frequency Networks (MBSFN) [13]. Smaller MBSFN networks distributed regional content and larger MBSFN networks distribute non regional programming. Both broadcast and unicast distribution method were included in the analysis, simply where unpopular content would be transmitted using unicast radio bearers. For broadcast only, the paper presented only limited saving in required bandwidth over DTV in rural environments with ISDs of $12 \mathrm{~km}$ or less. In urban environments where ISDs are $1.5 \mathrm{~km}$ or less, savings of up to $160 \mathrm{MHz}$ are shown, although, these figures are for fixed antennas and rely on "reasonably optimistic assumptions". The study concluded that, with shifted TV consumption patterns, "CellTV can be beneficial".

The idea of hybrid use of unicast and eMBMS services has also been explored in multiple forms. Albeit a different hybrid approach to the work proposed, Monserrat et al. in [14] use eMBMS services for unidirectional file delivery to multiple users. By providing a "post-delivery repair phase", any user unable to decode the file after broadcast transmission were successfully able to perform recovery via a pt-p bearer. More closely related, recent work in [15] uses a Joint Multicast/Unicast Scheduling strategy to overcome the aforementioned limitation of CMS catering for channel conditions of all users requiring service. Here, de la Fuente et al. performed an assessment at each LTE frame to establish a compromise on between the multicast and unicast groups.

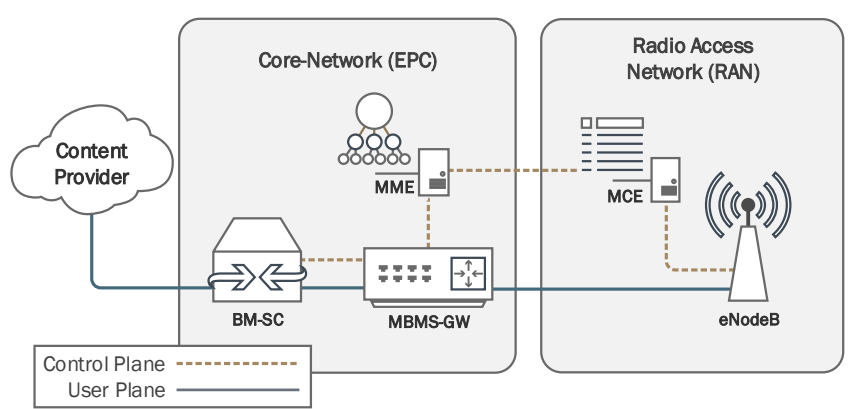

Fig. 2. LTE eMBMS logical architecture

The result of this assessment then dictates the allocation of resources to, and the MCS selection for, multicast services. The remaining resources are allocated back to the unicast scheduler.

\section{LTE EMBMS ARCHITECTURE}

The Multimedia Broadcast Multicast Service (MBMS) was first introduced with Universal Mobile Telecommunications System (UMTS) release 6, defined in 2003 [16]. Evolved for LTE, the MBMS standard became known as eMBMS, although conceptually existing since LTE's initial introduction in Release 8 (frozen in March 2009), implementation details were not specified until March 2010 with the freeze of release 9 [17]. As with CMS, eMBMS facilitates synchronous transmission to multiple users through shared use of the same radio resources. On the radio interface of the network, this is done by establishing a p-t-m radio bearer [17].

\section{A. eMBMS Architecture}

The overall LTE system architecture can be broadly split into two halves, the Evolved Packet Core (EPC) and the Radio Access Network (RAN). The EPC performs the management and routing tasks required by a mobile broadband network. This includes establishing end to end connections, user authentication, charging and policy re-enforcement [18]. The RAN contains primarily one type of node, the eNodeB. Its functionality includes Radio Resource Control (RRC) and Modulation and Coding Scheme (MCS) selection, Admission Control, Scheduling, Enforcement of Quality of Service (QoS) and of most interest to this work, management of eMBMS session control and bearer establishment [5], [18].

The LTE eMBMS architecture is shown in Figure 2 along with both user and control plane interconnections. Content providers will interface with the Broadcast Multicast Service Center (BM-SC) which establishes and manages the data flow configuration through the EPC. From here the IP stream is forwarded to the eMBMS Gateway (MBMS-GW) which manages the distribution of the stream of eMBMS data packets to each participating eNodeB via IP Multicast, efficiently using the backhaul network [18]. The MBMS GW is also responsible for handling the session control signalling of each eMBMS service which is performed via the MME that keeps a record 


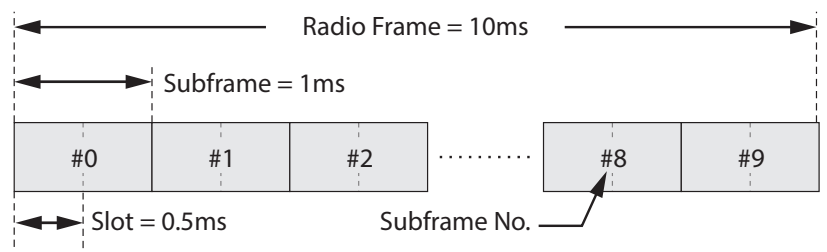

Fig. 3. Structure of frame type 1 (FDD) in downlink

TABLE I

OFDMA AND RESOURCE BLOCK PARAMETERS IN LTE

\begin{tabular}{|c|c|c|c|c|c|c|}
\hline Transmission BW (MHz) & $\mathbf{1 . 2 5}$ & $\mathbf{2 . 5}$ & $\mathbf{5}$ & $\mathbf{1 0}$ & $\mathbf{1 5}$ & $\mathbf{2 0}$ \\
\hline No. of Resource Blocks & 6 & 15 & 25 & 50 & 75 & 100 \\
\hline Sub-Carrier Spacing & \multicolumn{5}{|c|}{$15 \mathrm{KHz}$} \\
\hline Sub-Carriers per RB & \multicolumn{6}{|c|}{$180 \mathrm{KHz}\left(12^{*} 15 \mathrm{KHz}\right)$} \\
\hline (PRB) BW & \multicolumn{6}{c}{} \\
\hline
\end{tabular}

of UE properties, such as location, connected or idle status and is responsible for the setup and release of resources [5]. Connected to the MME, via the control plane, is the Multicast Coordination Entity (MCE), a key node for this research. This is a 'logical' entity, which is to say that it can be implemented as either a hardware node, or a software update in the LTE base station, referred to as the Evolved UMTS Terrestrial Radio Access Network (E-UTRAN) Node B or 'eNodeB' and sits within the RAN. The responsibilities of the MCE include the radio resource management of all eMBMS services for each of the connected eNodeBs, as well as decisions on MCS selection and frame allocation [5].

\section{B. Unicast and eMBMS Resource Allocation}

Both unicast and eMBMS are based on the Orthogonal Frequency Division Multiple Access (OFDMA) scheme for downlink data traffic. Despite this, the way in which each performs allocation of resources in both the frequency and time domain vary vastly.

LTE supports both Frequency and Time Division Duplexing (FDD and TDD) modes for downlink and uplink allocation with frame type 1 and 2 to represent each respectively [19]. The proposed work will only focus on the FDD type 1 frame structure, shown in Figure 3. Each frame has a duration of $10 \mathrm{~ms}$ and is formed of ten $1 \mathrm{~ms}$ subframes that are then further split into two $0.5 \mathrm{~ms}$ slots. Physical resource allocation in LTE is carried out using a unit called the Resource Block (RB) which covers one slot, $0.5 \mathrm{~ms}$ in time and twelve subcarriers, $180 \mathrm{KHz}$, in frequency [19]. Since the Transmission Time Interval (TTI) and time domain unit for LTE scheduling is set at $1 \mathrm{~ms}$, the minimum allocation unit is formed of two RBs and is called a Physical Resource Block (PRB). This structure is illustrated in Figure 4. As the available bandwidth is increased, more schedulable RBs are made available per time slot. Table I shows how many RBs are available per slot for each permitted LTE transmission bandwidth.

It is this granularity in both the time and frequency domains that provides unicast its spectral efficiency edge, allowing resource blocks to be scheduled depending on the channel conditions experienced by the UE in the current TTI.

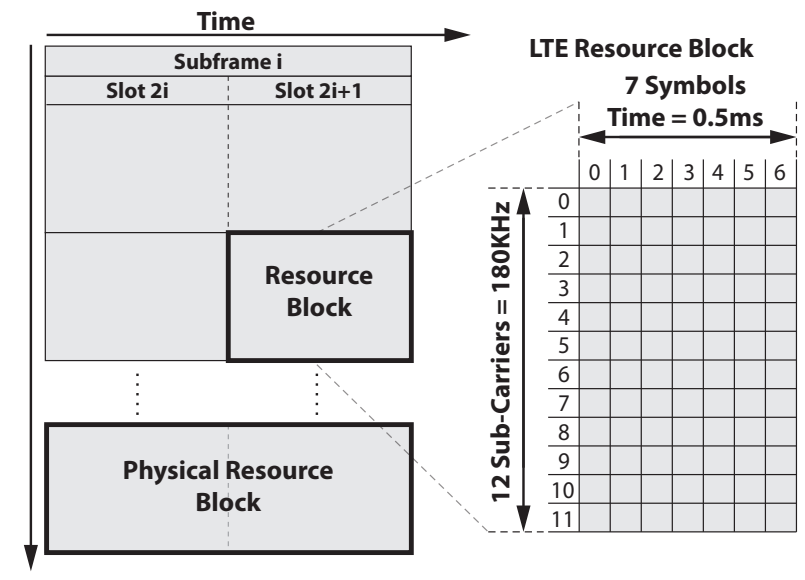

Fig. 4. Resource Block structure and allocation

For any eNodeB that provides an eMBMS service, certain frames are periodically allocated for the transmission of the Multicast Channel (MCH) [20]. Allocations can be made in two modes, 'oneFrame' where a single frame is allocated each time, or 'fourFrame' where allocation is in sets of 4 consecutive frames [21]. For the purposes of this explanation, 'oneFrame' based allocation will be assumed.

Ordinarily, no dynamic allocation of eMBMS resources is performed, instead frame reservation is based on the 'radioframeAllocationPeriod' and 'radioframeAllocationOffset' parameters. All radio frames that satisfy:

$$
S F N \bmod A p=A o
$$

are reserved for the eMBMS service, where $S F N$ is the current System Frame Number and $A p$ and $A o$ represent the chosen Allocation Period (AP) and allocation offset respectively [21]. An example presented in Figure 5 more clearly illustrates this allocation, where an offset of 2 and scheduling period of 4 are shown mapped over time.

Once a frame is reserved to contain eMBMS services, only six of the ten available subframes within can be used for the broadcast service. This is due to synchronisation and paging that can occupy subframes $0,4,5$ and 9 of any LTE Type 1 frame, making them unusable for eMBMS services [22]. In order to denote which subframes have been allocated within the reserved frame, a bitmap is used, each bit denoting true or false for an eMBMS or Unicast subframe assignment respectively. Only the subframes that may be allocated to eMBMS services are represented by the bitmap; therefore, a 6-bit map would represent 'oneFrame' allocation and a 24bit map would be utilised for 'fourFrame' allocation [22]. The example illustrated in Figure 5 uses bitmap '110101', therefore allocating all but subframe numbers 3 and 7 of the allocatable range to eMBMS. A reserved subframe utilises the entire bandwidth allocation in the frequency domain for its duration.

In order to decode the eMBMS data, the UE must know the allocation period and offset parameters, bitmap and MCS chosen to transmit data. This information is updated during each subframe and cannot be changed until an update is sent 


\begin{tabular}{|c|c|c|c|c|c|c|c|c|c|c|c|c|}
\hline \multirow[b]{2}{*}{ SFN } & \multirow[b]{2}{*}{ eMBMS } & \multicolumn{10}{|c|}{ Sub Frame \# } & \multirow{4}{*}{$\begin{array}{l}\text { Scheduling } \\
\text { Offset }=2\end{array}$} \\
\hline & & 0 & 1 & 2 & 3 & 4 & 5 & 6 & 7 & 8 & 9 & \\
\hline 0 & $\mathrm{~N}$ & & & & & & & & & & & \\
\hline 1 & $\mathrm{~N}$ & & & & & & & & & & & \\
\hline 2 & $Y$ & & & & X & & & & X & & & \multirow{7}{*}{$\begin{array}{l}\text { Scheduling } \\
\text { Period }=4\end{array}$} \\
\hline 3 & $\mathrm{~N}$ & & & & & & & & & & & \\
\hline 4 & $\mathrm{~N}$ & & & & & & & & & & & \\
\hline 5 & $\mathrm{~N}$ & & & & & & & & & & & \\
\hline 6 & $Y$ & & & & X & & & & X & & & \\
\hline 7 & $\mathrm{~N}$ & & & & & & & & & & & \\
\hline 8 & $\mathrm{~N}$ & & & & & & & & & & & \\
\hline
\end{tabular}

Sub Frame Bitmap [6 bit] : 110101

Unicast Subframe $\square$ eMBMS Subframe

XMBMS Capable Subframe but not mapped so assigned to Unicast

Fig. 5. LTE MBSFN frame \& subframe scheduling with MBSFN AP $=4$

on the control channel. The delay between these updates is also selectable and is named the "MCHSchedulingPeriod". Control channel update periods are limited to every $32,64,128$ or 256 frames, with shorter durations adding greater signalling overhead [21]. It is the loss of the fast and dynamic ability to schedule, as well as the need for the same transmission parameters to cater for a larger user base, that can lead to lower spectral efficiency if there is little interest in the broadcast content.

\section{Hybrid UNICAST BROADCAST SYNCHRONISATION FRAMEWORK}

The HUBS system's primary objective is to, despite varying cell conditions and loading, minimise the time offset between related streams delivered jointly by LTE unicast and broadcast services. By considering stream offset in addition to delay, the streams may adapt, together, to varying load conditions. Since bandwidth must be split between eMBMS and unicast services, this subsequently offers benefits to the entire cell. Consider a scenario where, due to insufficient unicast resources for the requested traffic, the stream begins to see an increased bearer queue building at the eNB. In this instance, the unicast stream will show a lag versus the broadcast, HUBS will then consider whether some resources from the broadcast stream can be freed and re-allocated to the unicast pool.

The LTE eMBMS architecture (presented in Section III-A), shows the MCE is uniquely positioned to gather the required user data. It is the entity that holds responsibility for radio resource management of all eMBMS services. It is therefore chosen as the key node in which to implement the HUBS framework management. A complete overview of the hubs dynamic allocation algorithm is presented in pseudocode in Algorithm 1. Within the MCE, a HUBS group $G_{I D}$ is created for each eMBMS service offering enhanced streams via unicast. For each $G_{I D}$, a set of users $K=\left\{x \in \mathbb{Z}^{+} \mid x \leq N\right\}$ is maintained where, $N$ is the total number of users requesting an enhancement stream. Considering that LTE does not allow the changing of parameters for a given eMBMS service without first issuing an update on the control channel, there is a limit on how frequently the resource allocation of the service can be dynamically varied. Therefore, each group defines a dynamic allocation period $P_{d a}$ in LTE system frames, where $P_{d a} \in$ $\{32,64,128,256\}$ and $P_{d a}>M C H S c h e d u l i n g$ Period. Given the frequent variability of cell conditions as well as instantaneous nature of video stream bitrate (i.e., size of an I versus a $P$ frame), it is inadequate to make an assessment of stream offset and subsequent dynamic allocation decision based on only a single time instance. Therefore, an average is maintained within each HUBS group by sampling the offset with greater frequency between each dynamic allocation decision. Let $P_{s p}$ LTE system frames define this more frequent period, where $P_{s p} \in\{4,8,16,32\}$. The number of samples $S$ contributing to the dynamic decision will therefore be given by $P_{d a} / P_{s p}$. During sampling within a group, each member user $k$ has the time offset $\Delta_{k}$ between their corresponding unicast and broadcast streams calculated by probing the bearer queues at the eNB. A positive or negative $\Delta_{k}$ implies the broadcast stream is leading or lagging respectfully. The broadcast stream is therefore defined as the anchor with which the offset of each unicast stream will be measured. In the case where the broadcast and unicast streams are transmitting the same video frame, we set $\Delta_{k}$ to zero.

To improve accuracy in the case where the broadcast stream leads the unicast, a record of the previous 10 video frame numbers, along with their transmission times is kept for the broadcast stream. Since transmission time will vary dependant on the size of the current video frame to be transmitted, storing the previous values allows an exact offset calculation to be made. Where the offset $\Delta_{k}$ is to be calculated, the time of transmission of the current unicast video frame number, $f$ of user $k$ is subtracted from the stored transmission time of frame number $f$ of the broadcast. When the calculation is made, since $f$ is the frame currently being transmitted by the unicast, the time for this frame can be taken as $t_{\text {now }}$, the current simulator time, thus producing the equation:

$$
\triangle_{k}=t_{\text {now }}-t b_{f}
$$

where $t b_{f}$ equals the time of transmission within the broadcast stream of frame number $f$. This is of course only available for the preceding 10 frames (i.e. where $F_{b}$ is the current video frame being broadcast $\left.t b_{f} \forall f \in\left\{x \in \mathbb{Z}^{+} \mid F_{b}-10<x \leq F_{b}\right\}\right)$. Maintaining a similar updated record for each unicast user in the group would prove computationally expensive. Therefore, only the current video frame $F_{u, k}$ of the unicast stream for user $k$ is retrieved.

Should a unicast user lead the broadcast stream, the stream offset will be calculated by establishing how many frames the lead consists of, multiplied by the frame duration:

$$
\triangle_{k}=j\left(F_{b}-F_{u, k}\right)
$$

where $j$ is the video frame duration in milliseconds. Once the stream offset for each user within the HUBS group is calculated, an average, $\triangle_{a v e, i}$ is taken where $i \in\left\{x \in \mathbb{Z}^{+} \mid 0<x<S\right\}$. This results in $S$ averaged samples having been collected during the current dynamic allocation period. When a dynamic allocation decision is requested, 
it will be based on the average offset of the group given by:

$$
\triangle_{D e c}=\frac{1}{S} \sum_{k=1}^{S} \triangle_{a v e, k}
$$

The algorithm now makes a decision based on the group's average offset $\triangle_{D e c}$ value. Should $\triangle_{D e c}$ drift below or above $10 \%$ of the maximum delay defined within the QoS conditions on the broadcast bearer, the algorithm will increase or decrease resources reserved for broadcast accordingly. The HUBS algorithm will also honour the broadcast bearers QoS constraints for maximum delay, guaranteeing service conditions for broadcast only users who are not members of the HUBS group.

\section{Simulation Scenarios}

This paper presents two simulated scenarios exploring multi-stream multimedia use cases. Each scenario is designed to evaluate different aspects of the HUBS framework's ability to dynamically respond to varied conditions. The two scenarios are as follows:

\section{A. Scenario A: Interactive Companion View}

In this scenario, the primary coverage of a football match is broadcast within a cell to all subscribing users. The pseudo broadcaster then additionally offers a multitude of fixed views and streams that the user can interactively select between to accompany the main broadcast. In the simulation, there is a total of 8 additional views available, one of which is randomly selected by each user requesting a companion view at the start of each simulation run as illustrated in Figure 6. The sequences provided by the TRICTRAC ${ }^{1}$ project comprise synthetic views of a football match from various angles around the stadium. Each view is encoded using the H.264/AVC video codec at a resolution of $1280 \times 720$ pixels [23]. The enhancement sequences being unicast see minimal bitrate variation for their duration but vary significantly amongst one another. This is expected given the different angles and varied focal lengths. The data rates of the videos are shown in Figure 7. In this set of sequences, after encoding, the primary broadcast stream has a lower average bitrate than the unicast enhancement options. This is an interesting real world possibility and considering the random selection of media will do well to test the frameworks response.

\section{B. Scenario B: H.264/AVC MVC encoded Stereoscopic 3D}

This scenario explores the delivery of a popular live television event with stereoscopic 3D coverage (i.e., a football game or a motorsport event). The left view of the stereoscopic pair is encoded independently using the MVC extension of H.264/AVC [24]. The result is a stream that can be decoded without any additional information by any H.264/AVC compliant decoder. The right view is encoded using the MVC extensions ability to code dependently on the left view, making use of redundant information present in both to reduce the stream size. In this case, users wishing to decode the right

\footnotetext{
${ }^{1}$ Production: TRICTRAC Project: http://www.multitel.be/trictrac
}

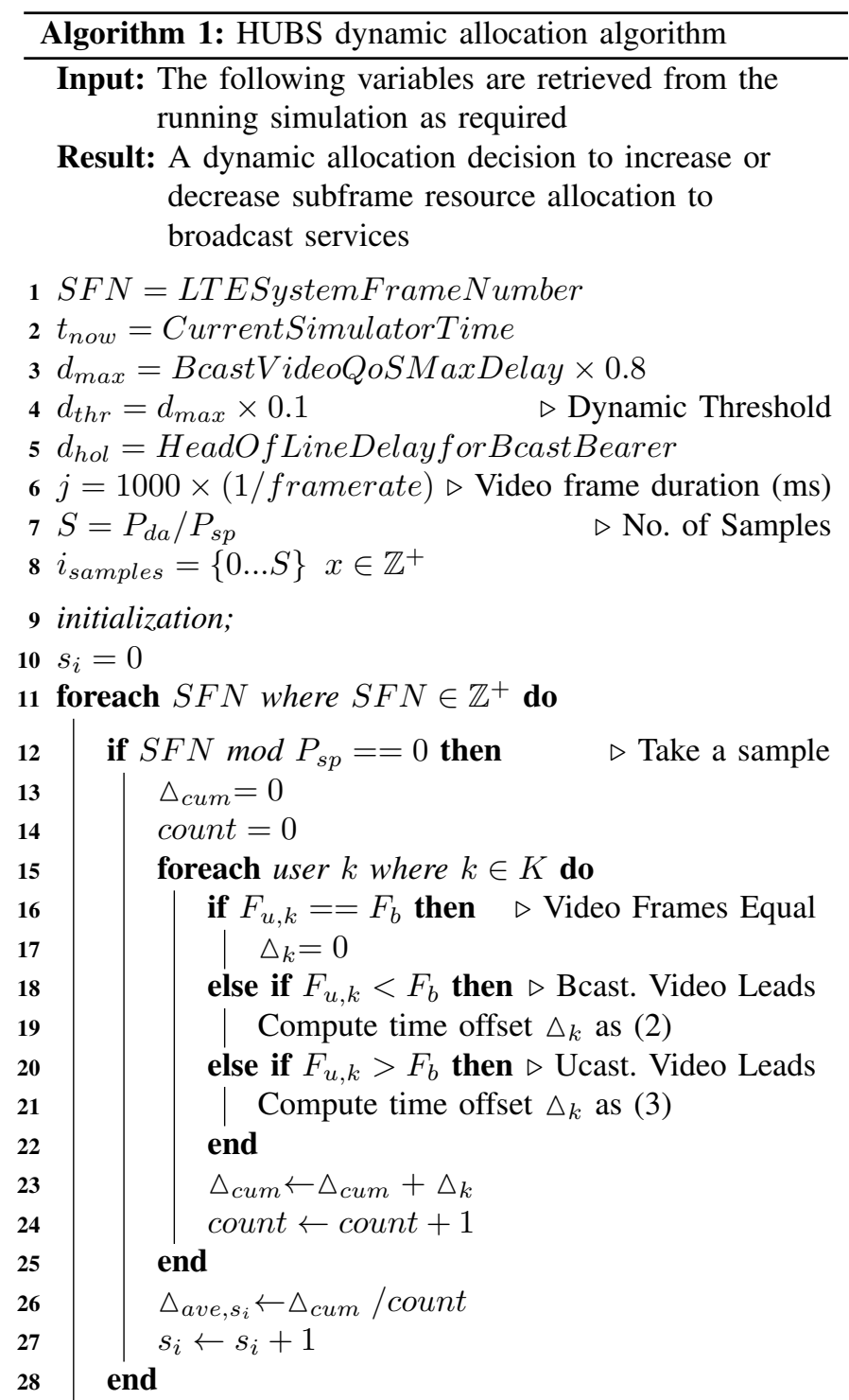

view must be receiving the left view simultaneously. The left view is broadcast to all subscribing users within the cell. Since it is likely only a subset of users within the cell will be capable of, or choose to watch the coverage in $3 \mathrm{D}$, these users are 


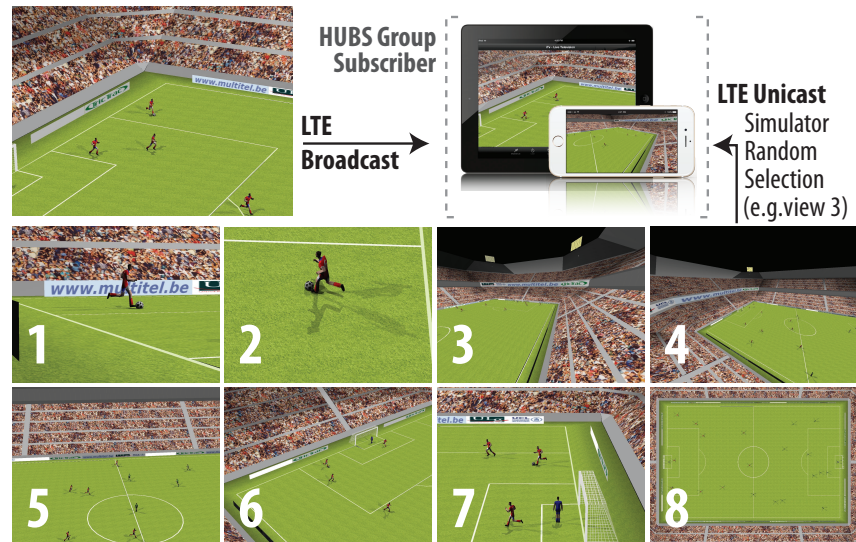

Fig. 6. Interactive companion view scenario A diagram

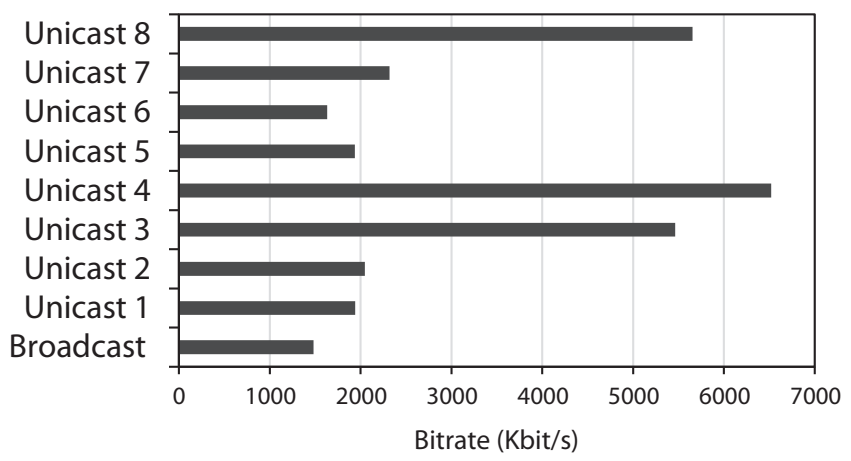

Fig. 7. Variation of encoded bitrates of scenario A video sequences

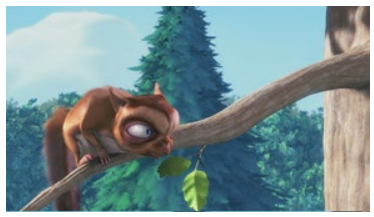

(Left View) LTE Broadcast

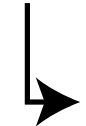

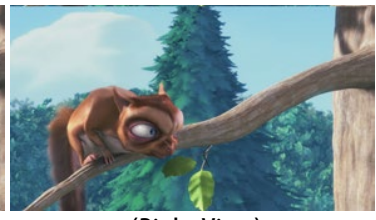

(Right View)

LTE Unicast

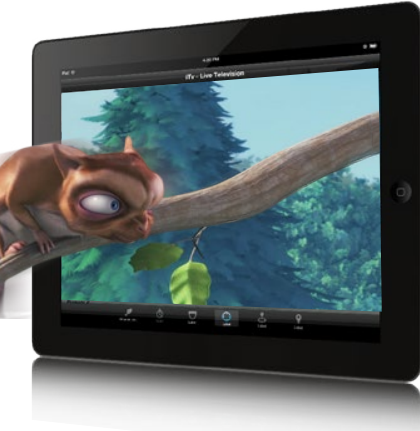

HUBS

Group

Subscriber

Fig. 8. H.264/AVC MVC encoded Stereoscopic 3D scenario B diagram

catered for using unicast transmissions.

This scenario provides a particular focus on how effectively the dynamic allocation of the HUBS framework responds to variations of video content during the broadcast. Given the content has a significant impact on the video encoder and resultant data rate, in order to best test the proposed model, a video sequence with properties true to a typical live broadcast was created. This sequence was formed of multiple test clips
TABLE II

BREAKDOWN OF COMPILED SCENARIO B VIDEO SEQUENCE

\begin{tabular}{|c|c|c|c|c|}
\hline & \multicolumn{3}{|c|}{ Original Sequence Frames } & Duration \\
New Clip Name & Start & End & Total & Seconds @ 25fps \\
\hline 24h Clip 1 & 1320 & 1820 & 500 & 20 \\
\hline 24h Clip 2 & 2500 & 3000 & 500 & 20 \\
\hline Big Buck Bunny & 10500 & 11500 & 1000 & 40 \\
\hline Café Cam 4+5 & 0 & 250 & 250 & 10 \\
\hline Poznan St Cam 4+5 & 0 & 250 & 250 & 10 \\
\hline Shark Cam 4+5 & 0 & 250 & 250 & 10 \\
\hline Soccer Cam 4+5 & 0 & 250 & 250 & 10 \\
\hline Total & - & - & $\mathbf{3 0 0 0}$ & $\mathbf{1 2 0}$ \\
\hline
\end{tabular}

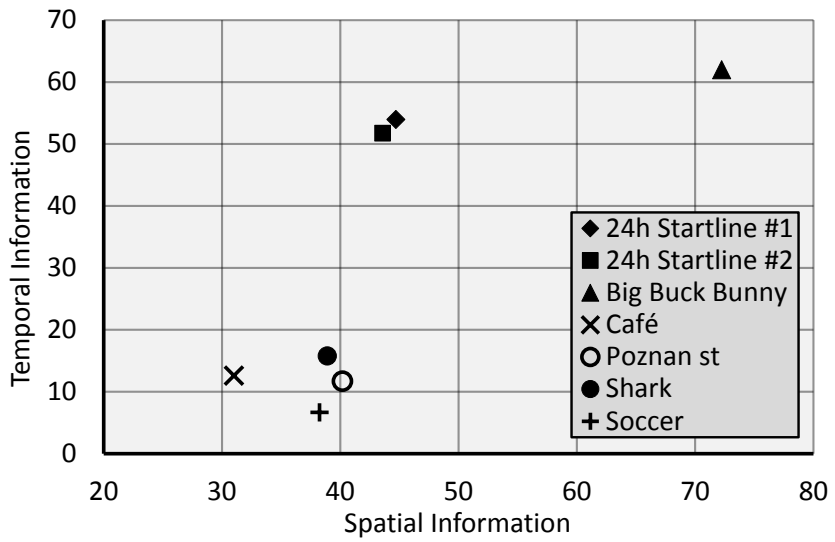

Fig. 9. Spatial and Temporal Index of chosen scenario B sequences

compiled from sequences available and familiar to the research community. Each of these has been chosen to provide a mix of both spatial and temporal information more representative of a live video broadcast. This included fixed and panning camera shots as well as scene cuts. Table II lists, in order, the name and duration of each clip used to compile the final sequence totalling 120 seconds run time.

In order to draw objective comparisons of both spatial detail and temporal change information, and hence the coding difficulty, analysis was performed on each clip. Spatial perceptual Information (SI) was measured based on the Sobel filter utilising the method defined in [25]. For Temporal perceptual Information (TI) standard deviation is performed across each pixel of the current frame with the prior also using the method defined in [25]. For both SI and TI, the maximum value in the time series is taken as the representative value. The results for each clip are shown in Figure 9.

Screenshots from each clip are displayed in Figure 10. The first two clips used are excerpts from the "24h" [26] racing sequence featuring high action car racing content with frequent scene cuts. The following clip is an action scene taken from "Big Buck Bunny" [27], an animated sequence that is high in both action, texture and detail. Figure 9 confirms visual observations placing these clips at the higher end of the Temporal and Spatial information scales. Café ${ }^{2}$ represents a typical fixed camera indoor studio soap opera style scene and Poznan Street $^{3}$, also fixed camera, represents a typical outdoor

\footnotetext{
${ }^{2}$ Production: Gwangju Institute of Science and Technology (GIST)

${ }^{3}$ Production: Poznan University of Technology
} 


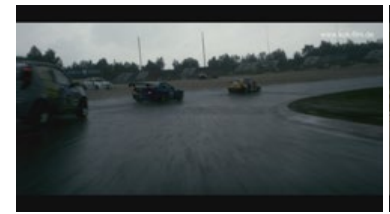

24h Clip 1

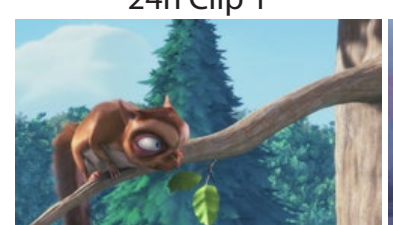

Big Buck Bunny

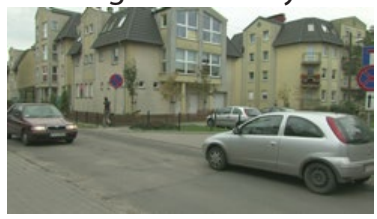

Poznan St

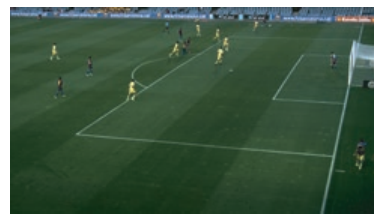

Soccer

Fig. 10. Screenshots of each clip compiled to form scenario B video sequence

scene with object motion. Shark ${ }^{4}$ is an animated underwater scene with a moving camera and moderate motion. Finally Soccer ${ }^{5}$ is fixed camera coverage of a soccer game within a stadium.

\section{Vi. Performance Evaluation}

In order to evaluate the proposed HUBS framework, an implementation has been built upon the open source system level LTE-Sim platform [28]. The LTE-Sim platform is widely used and accepted amongst the scientific community, making it a logical choice for the implementation of the HUBS framework. Originally, the simulator did not support eMBMS functionality and thus this functionality was also added before the HUBS framework could be established.

For each scenario, identical simulations are run with and without the HUBS dynamic allocation framework enabled. Where no dynamic allocation is utilised, the simulation is run for all possible subframe bitmaps. Each simulation is iterated through 10 runs, each with a new random seed. Every run has a total simulation duration of 150 seconds, consisting of a warm up period of 30 seconds followed by 120 seconds of application data transmission. The core parameters of each simulation remain fixed between each scenario, these are shown in Table III along with assignment percentages of user services across the cell. Given the cell is transmitting multiple HD video content streams, the highest available bandwidth of $20 \mathrm{Mhz}$ is selected. The cell layout surround the active cells with addition interference generating cells, ensuring realistic

\footnotetext{
${ }^{4}$ This sequence is provided by NICT for MPEG FTV standardization.

${ }^{5}$ Production: FINE - Free-viewpoint Immersive Networked Experience Project.
}

TABLE III

SIMULATION PARAMETERS

\begin{tabular}{|c|c|}
\hline Parameter & Value \\
\hline Bandwidth & $20 \mathrm{MHz}$ \\
\hline Number of RBs & 100 \\
\hline Simulation Time & 150s Per Run, 30s Warm Up, 10 Runs \\
\hline Cells & Simulation takes place in a single cell \\
\hline Cell Layout & $\begin{array}{l}\text { Hexagonal grid of } 7 \text {. Surrounding cells } \\
\text { generate interference }\end{array}$ \\
\hline User distribution & $\begin{array}{l}\text { Random Placement, walking in random } \\
\text { direction }\end{array}$ \\
\hline User Numbers & $5-40$ users, interval of 5 \\
\hline eMBMS AP & 1 frame \\
\hline eMBMS SF Allocation & 1 frame \\
\hline $\begin{array}{l}\text { eMBMS SF Bitmaps } \\
\text { (Static Allocation) }\end{array}$ & $\begin{array}{l}111111,111110,111100,111000, \\
110000,100000\end{array}$ \\
\hline eMBMS MCS Index & 8 \\
\hline Acknowledge Mode & Disabled \\
\hline Frequency Reuse & Enabled (3 Clusters) \\
\hline Channel Realization & Macro Cell Urban Area \\
\hline Error Model & Wideband CQI Eesm Error Model \\
\hline Link Adaptation & AMC Enabled \\
\hline $\begin{array}{l}\text { Unicast Scheduling } \\
\text { Algorithm }\end{array}$ & $\begin{array}{l}\text { Maximum-Largest Weighted Delay } \\
\text { First (M-LWDF) }\end{array}$ \\
\hline QoS Max Delay & $\begin{array}{l}\text { eMBMS }=100 \mathrm{~ms} \\
\text { Video }=100 \mathrm{~ms}(\mathrm{QCI}-7) \\
\text { VoIP }=100 \mathrm{~ms}(\mathrm{QCI}-1)\end{array}$ \\
\hline $\begin{array}{r}\text { User Service } \\
\text { Broadcast Video } \\
\text { Enhanced Video } \\
\text { Standalone Video } \\
\text { Voice Calls } \\
\text { Internet Browsing }\end{array}$ & $\begin{array}{l}60 \% \text { Total Active Users } \\
50 \% \text { Broadcast Subscribers } \\
10 \% \text { Total Active Users } \\
20 \% \text { Total Active Users } \\
10 \% \text { Total Active Users } \\
\text { (Where a non integer number results, } \\
\text { number of users always rounded down.) }\end{array}$ \\
\hline
\end{tabular}

channel conditions for edge users. Users are assigned positions and directions generated randomly using a pre-established seed which changes for each run of the simulation.

\section{A. Performance in Scenario A: Interactive Companion View}

For analysis, results for this scenario have had bitmap '100000' removed as, with so little resource allocation, the broadcast service is unable to sustain any part of the video bit stream. It therefore would not have been a viable option for transmission of the selected video.

The first objective for evaluation is the Inter-stream Arrival Difference (IAD); the difference in arrival time measured in milliseconds between the joint unicast and broadcast streams. The IADs are extracted from the completed simulation trace files. By reconstructing each video frame from the received LTE radio frames, the time that a completed video frame was received can be established for each service for a given user. The difference is then averaged across the simulation runs and users. The proposed HUBS dynamic allocation framework shows clear minimisation of IAD with increased gain in a more heavily loaded cell, see Figure 11(a).

A threshold value $d_{t h r}$ is defined as $10 \%$ of eMBMS QoS Max Delay, therefore a value of $\pm 10 \mathrm{~ms}$ is taken by the HUBS algorithm (See Section IV). Although some variation from zero can be observed, the IAD sits comfortably within the threshold defined, which implies that the streams were synchronised without seriously approaching their respective QoS 
(a)

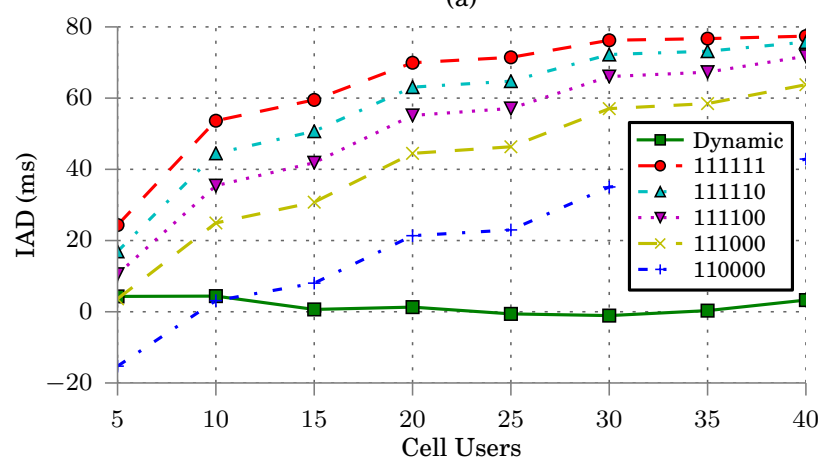

(c)

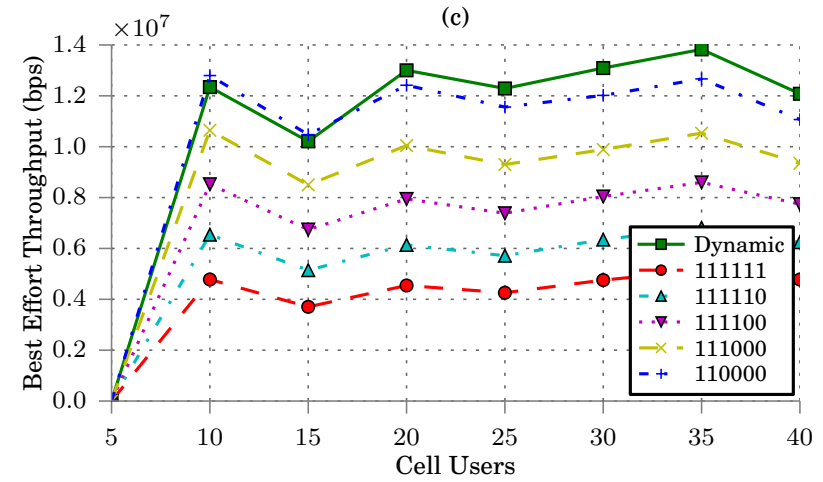

(b)

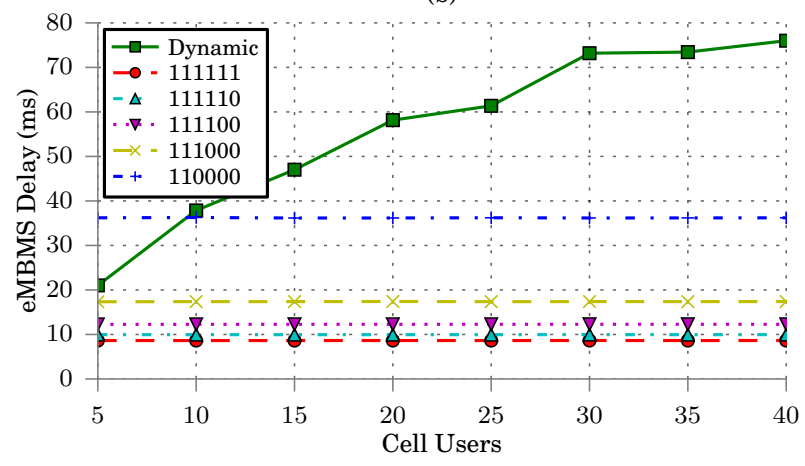

(d)

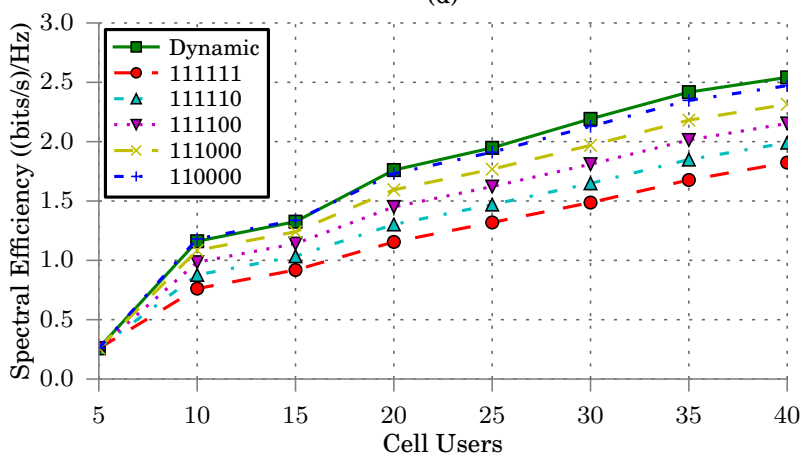

Fig. 11. Scenario A: (a) Unicast-Broadcast Inter-stream Arrival Difference (IAD), (b) eMBMS Service Delay, (c) Best Effort Service Cell Throughput, (d) Overall Cell Spectral Efficiency.

delay limitations. This is further confirmed by Figure 11(b) that shows the HUBS algorithm increasing eMBMS delay as unicast services cause the cell to become more congested. The flattening off of the curve just below $80 \mathrm{~ms}$ is due to the HUBS algorithm refusing to further reduce resource allocation to the broadcast stream as demonstrated by the conditions in, Algorithm 1 lines 34 and 37. Furthermore, dynamic allocation saw an average Packet Loss Rate (PLR) difference of less that $\pm 0.13 \%$ with any of the remaining static bitmap allocations for the broadcast stream.

With dynamic allocation enabled, all real-time services, including VoIP and independent video services within the cell remained commensurate with, or surpassed the performance of even the highest performing static allocation map. Shown in Figure 11(c), the non-real-time best effort service that emulates typical web browsing services within the cell showed a marked increase in throughput with increased congestion. Some traces in Figure 11 exhibit some variation in the trace between cell user increases, most visible in (c) between 10 and 25 users. This is due to the method in which the percentage of user services defined in Table III are translated into users with assigned services in the simulation scenario. Taking 10 and 15 cell users as an example, 5 more users are requested from the simulator but this will translate to 3 more broadcast users, 1 more enhanced user, 1 more VoIP user and the same number of users for standalone video and internet browsing services. Given services such as best effort do not see an increase in users where others do, this will cause the variations in results shown.

When the cell becomes congested, freeing up non essential resources allows more efficient instantaneous use of spectrum by the unicast schedulers. Consequently, an increase in overall cell spectral efficiency can also be observed in Figure 11(d).

\section{B. Performance in Scenario B: H.264/AVC MVC encoded Stereoscopic $3 D$}

This scenario explores how the HUBS Dynamic Allocation responds to varying video content often seen in live broadcast coverage. Here the broadcast stream closely follows the enhancement stream as they are a stereoscopic pair, sharing the same characteristics over time. This results in simultaneous peaks in data rate across both services introducing new challenges to the HUBS Framework. This can be clearly observed in Figure 14 (a), showing the frame sizes of the left and right streams. Figure 14 (b) illustrates the dynamic allocation decisions made by the framework for the selected Subframe Index (SI). The SI simply corresponds to the quantity of subframes allocated to broadcast over unicast (e.g., SI of 1 corresponds to map '100000', SI of 2 to '110000' etc.). Dynamic allocation can clearly be observed mapping SI to the video frame sizes as required, then releasing the resources where video bitrate drops. Given the greater peak data rate required over scenario $\mathrm{A}$, to support the full $\mathrm{HD}$ video being broadcast, static allocation of greater than 3 subframes in every six was required. Therefore, only bitmaps ' 111100 ', ' 111110 ' and ' 111111 ' have been included for analysis. 
(a)

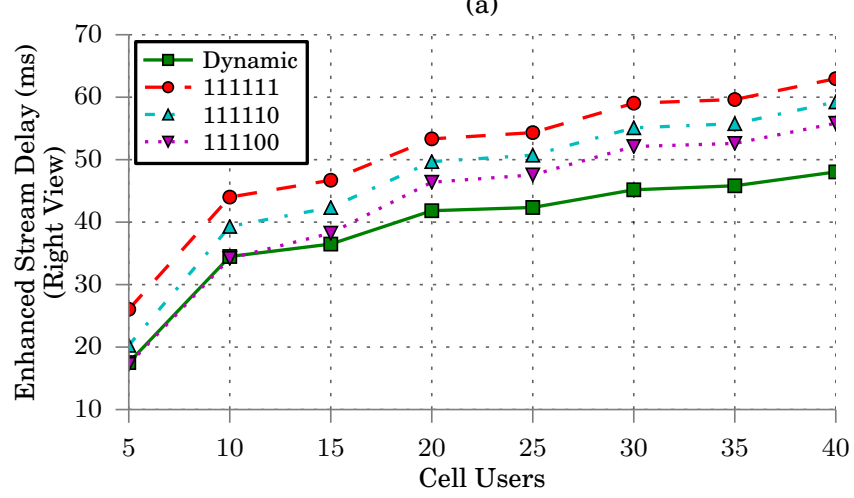

(c)

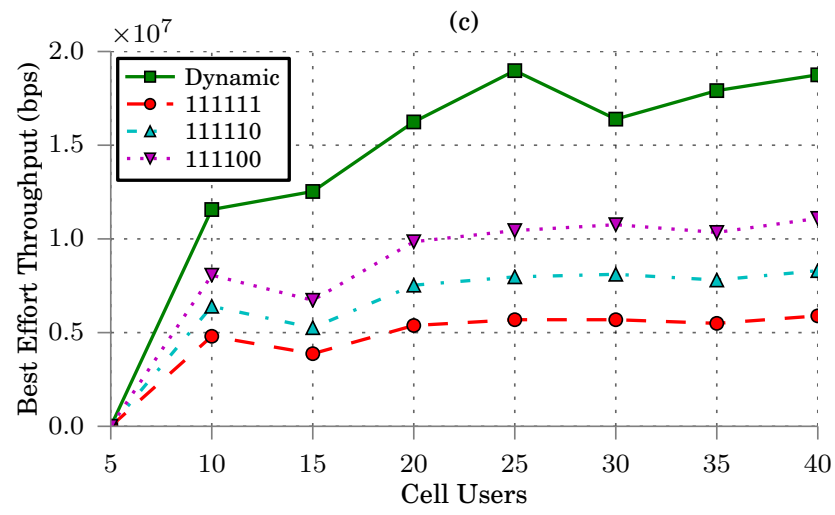

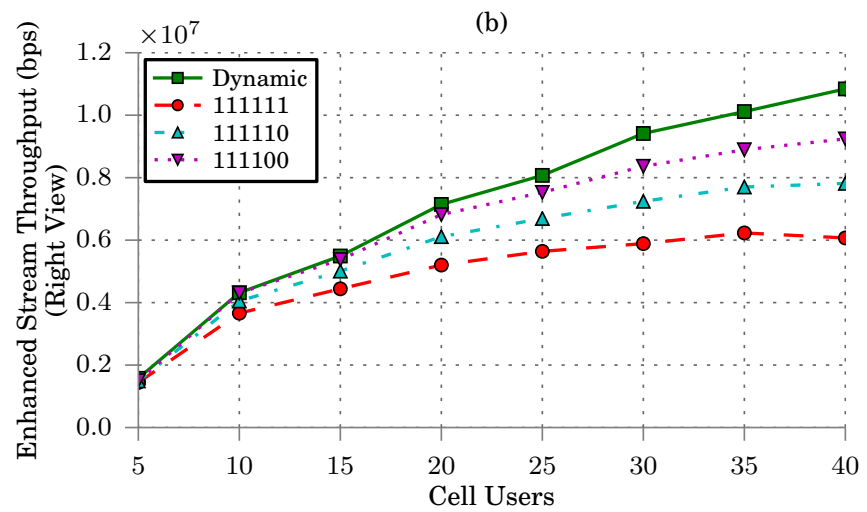

(d)

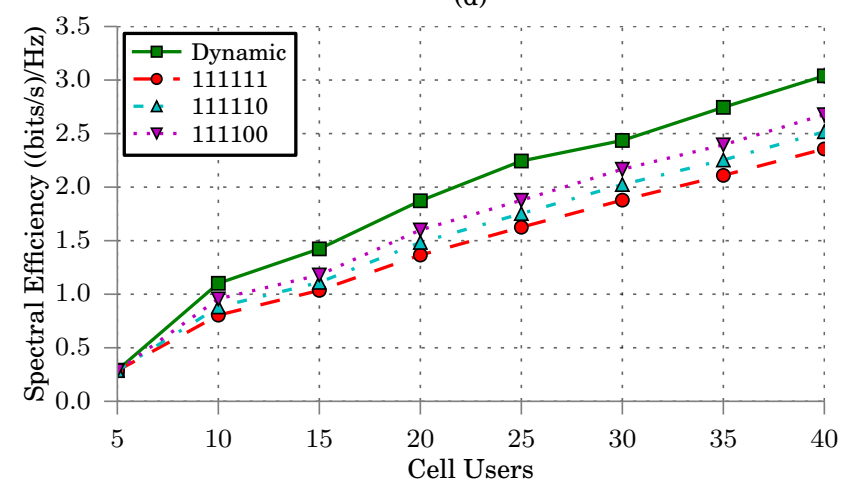

Fig. 12. Scenario B: (a) Unicast Enhancement Stream (Right View) Delay, (b) Unicast Enhancement Stream (Right View) Throughput, (c) Best Effort Service Cell Throughput, (d) Overall Cell Spectral Efficiency.

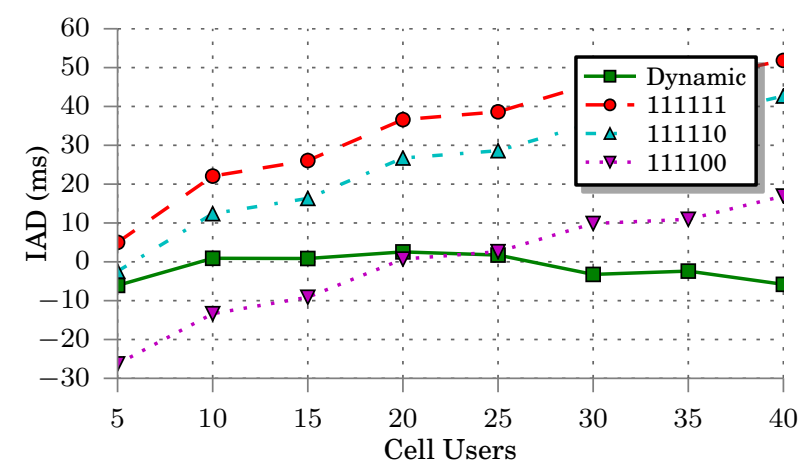

Fig. 13. Scenario B: Unicast-Broadcast Inter-stream Arrival Difference (IAD).

As with IAD in scenario A, the dynamic allocation framework successfully minimises the average between the streams, see Figure 13. Despite cell loading, the IAD is reduced to within the $\pm 10 \mathrm{~ms}$ threshold. Moreover, there is a negligible average PLR difference between the dynamically allocated broadcast stream and each static allocation, remaining within $\pm 0.8 \%$. The delay incurred to the broadcast stream does not exceed an average of $80 \mathrm{~ms}$ and only approaches this value when the cell is heavily loaded. This keeps the broadcast stream within its QoS max delay requirements whilst allowing more opportunistic allocation of resources with the unicast scheduler. With such an application, any loss in frame synchronisation presented to viewer is highly noticeable. As such, even though the IAD of the streams has been minimised to well within a single frame, minimal buffering of several frames at the receiver can guarantee synchronous playback.

By relaxing allocation of broadcast resources in certain instances, dynamic allocation allows for all unicast services within the cell to benefit, including the linked unicast video stream. A reduction in delay of this enhanced stream further minimises the IAD. This is shown in Figure 12 (a) along with an increase in throughput, shown in Figure 12 (b) allowing further users to be supported.

Non-real-time services with far less competitive resource allocation priorities see the largest improvements. Figure 12 (c) shows a significant improvement in the throughput of emulated web browsing traffic using the best effort service. Given both the broadcast and unicast video streams will vary in bitrate together, non-real-time services are able to utilise periods where resources are freed by dynamic allocation.

The overall spectral efficiency of the cell, illustrated in Figure 12 (d) sees a significant increase over any of the viable static allocation bitmaps. Across the range of cell loads, dynamic allocation has allowed for an increase of greater than $13 \%$ in the average spectral efficiency over the best performing '111100' static allocation. 
(a)

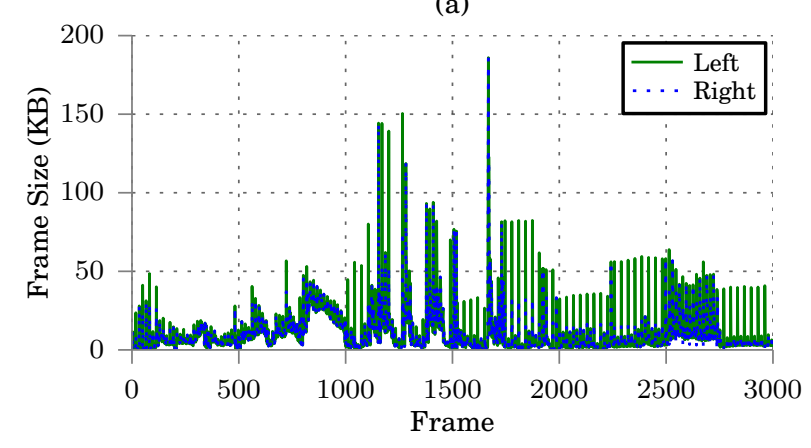

(b)

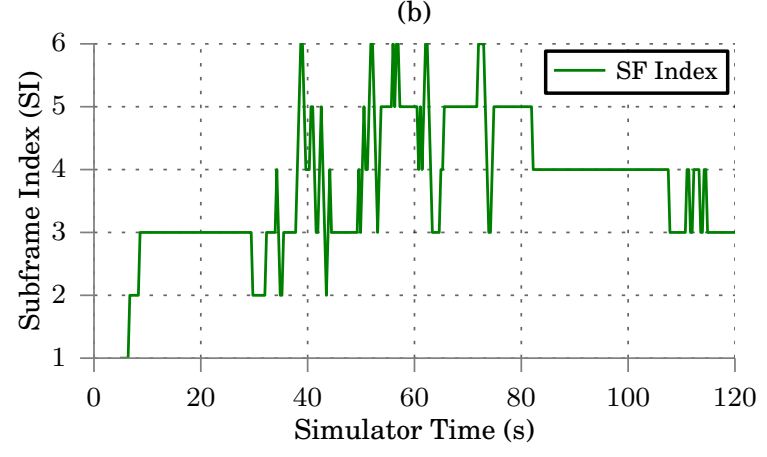

Fig. 14. Scenario B: (a) Video Frame Size for Left (Broadcast), Right (Enhanced) views, (a) Dynamically allocated Sub Frame Index (20 Cell Users).

\section{CONCLUSION}

This paper proposes a novel Hybrid Unicast Broadcast Synchronisation (HUBS) Framework targeted at meeting the challenges of delivering next generation multi-stream multimedia services within LTE networks, including: UHDTV, 3D Television, Free Viewpoint Television (FVT) and Multi/Companion Screen viewing. Simulated implementation and testing of such scenarios clearly demonstrated successful minimisation of inter-arrival time differences to within $10 \%$ of the LTE defined QoS delay specification. Not only are the streams brought into synchronisation, but this leads to an overall increase in overall cell spectral efficiency by allowing the more spectrally efficient LTE unicast scheduler increased resources during periods where the broadcast stream does not immediately require them. Additionally, with dynamic allocation enabled, improvements are observed across all other unicast cell services, surpassing the performance of any available static allocation map.

Importantly for mobile network providers, the proposed framework incurs minimal computational overhead and the required processing scales linearly with users. By conforming to the standards defined for eMBMS operation within LTE, the proposed framework may be implemented virtually, as a software upgrade within the Multicast Coordination Entity of existing or future LTE networks. Furthermore, the HUBS framework presented can provide network operators with a fast and automated method of assigning broadcast allocation parameters for any broadcast streams on the fly, these would have otherwise had to be defined prior to transmission.
Future work might explore the concept of utilising interstream arrival delay with content adaptation using Dynamic Adaptive Streaming over HTTP (DASH). This will allow the HUBS framework to dynamically scale the enhancement services delivered via unicast alleviating load at greater user numbers. Additionally, functionality allowing a popular enhancement stream to be moved from unicast delivery to a newly established eMBMS service seamlessly could benefit the framework in peak popularity scenarios. Furthermore, the HUBS framework lends itself well as a platform to implement a slow AMC capability to eMBMS streams in its control. Here, refreshing the MCS of a given eMBMS service could be done at each dynamic allocation period based on the CQI feedback of subscribed users.

\section{REFERENCES}

[1] CISCO, "Cisco Visual Networking Index : Global Mobile Data Traffic Forecast Update 2013 to 2018," 2014. [Online]. Available: http://www.cisco.com/c/en/us/solutions/collateral/service-provider/ visual-networking-index-vni/white $\backslash$ _paper $\backslash$ _c11-520862.html

[2] IGR, "Content for All - The Potential for LTE Broadcast / eMBMS White Paper," Tech. Rep. January, 2013.

[3] L. Electronics, "LG s View on Evolution of LTE in Release 12 and beyond," Tech. Rep. June, 2012.

[4] G. K. Walker, J. Wang, C. Lo, X. Zhang, and G. Bao, "Relationship Between LTE Broadcast/eMBMS and Next Generation Broadcast Television," IEEE Transactions on Broadcasting, vol. 60, no. 2, pp. 185-192, Jun. 2014.

[5] ETSI TS 36.300 REL-9 - E-UTRAN; Overall description; Stage 2, 3GPP Standard 36.300, 2013.

[6] M. Tanimoto and T. Fujii, "ISO/IEC JTC1/SC29/WG11 M11259 FTV (free viewpoint television): achievements and challenge," ISO/IEC, Tech. Rep. October, 2004.

[7] L. Zhang, Y. Cai, Z. He, C. Wang, and P. Skov, "Performance Evaluation of LTE MBMS Baseline," 2009 5th International Conference on Wireless Communications, Networking and Mobile Computing, no. 1, pp. 1-4, Sep. 2009.

[8] G. Araniti and M. Condoluci, "Adaptive resource allocation to multicast services in LTE systems," IEEE Transactions on Broadcasting, vol. 59 , no. 4, pp. 658-664, 2013.

[9] O. B. Karimi and J. Liu, "Power Efficient High Quality Multimedia Multicast in LTE Wireless Networks," in 2011 IEEE Eighth International Conference on Mobile Ad-Hoc and Sensor Systems. IEEE, Oct. 2011, pp. 161-163.

[10] M. Crussière, C. Douillard, S. Member, C. Gallard, M. L. Bot, B. Ros, A. Bouttier, and A. Untersee, "A Unified Broadcast Layer for Horizon 2020 Delivery of Multimedia Services," IEEE Transactions on Broadcasting, vol. 60, no. 2, pp. 193-207, 2014.

[11] ETSI EN 302755 Digital Video Broadcasting (DVB) Frame structure channel coding and modulation for a second generation digital terrestrial television broadcasting system (DVB-T2), ETSI Standard 302755 , 2012.

[12] L. Shi, E. Obregon, K. Sung, J. Zander, and J. Bostrom, "CellTV-On the Benefit of TV Distribution Over Cellular Networks: A Case Study," IEEE Transactions on Broadcasting, vol. 60, no. 1, pp. 73-84, 2014.

[13] ETSI TS 25.356 REL-11 - Introduction of the Multimedia Broadcast / Multicast Service (MBMS) in the Radio Access Network (RAN); Stage 2, 3GPP Standard 25.356, 2012.

[14] J. Monserrat, "Joint Delivery of Unicast and E-MBMS Services in LTE Networks," IEEE Transactions on Broadcasting, vol. 58, no. 2, pp. 157167,2012

[15] A. D. Fuente, A. G. Armada, and P. Raquel, "Joint Multicast / Unicast Scheduling with Dynamic Optimization for LTE Multicast Service," in European Wireless 2014 Joint, 2014, pp. 462-467.

[16] ETSI TR 21.902 REL-6 - Evolution of 3GPP system, 3GPP Standard 36.902, 2003.

[17] ETSI TS 23.246 REL-9 - Multimedia Broadcast/Multicast Service (MBMS) Architecture and functional description, 3GPP Standard 36.246, 2012.

[18] D. Erik, P. Stefan, and S. Johan, 4G LTE/LTE-Advanced for Mobile Broadband. Academic Press, 2011, no. 978-0-12-385489-6. 
[19] ETSI TS 36.211 REL-11 - E-UTRA; Physical Channels and Modulation, 3GPP Standard 36.211, 2013.

[20] E. Dahlman, S. Parkvall, and J. Sköld, 4G LTE/LTE-Advanced for Mobile Broadband, 2nd ed. Academic Press, 2011

[21] ETSI TS 36.331 REL-10 - Radio Resource Control (RRC); Protocol specification, 3GPP Standard 36.331, 2013.

[22] ETSI TS 36.443 REL-9 - E-UTRAN; M2 Application Protocol (M2AP), 3GPP Standard 36.443, 2010.

[23] M. C. Dolby Laboratories Inc., Fraunhofer-Institute HHI, "H.264/MPEG-4 AVC Reference Software Manual," 2009. [Online]. Available: http://iphome.hhi.de/suehring/tml

[24] R.-W. T. H. R. Aachen, "MVC Software Manual JMVC 8.5," 2008. [Online]. Available: https://mvclab.googlecode.com/files/ SoftwareManual.doc

[25] ITU, "RECOMMENDATION ITU-R BT.1788 - Methodology for the subjective assessment of video quality in multimedia applications," Tech. Rep., 2007.

[26] KUK-Film. (2011) 24h Race Nuerburgring. [Online]. Available: http:// www.kuk-film.de/en/referenzen.php?mehr=24h \_rennen $\backslash \# 24 \mathrm{~h} \backslash$ rennen

[27] B. Foundation. (2008) Big Buck Bunny. [Online]. Available: http: //www.bigbuckbunny.org/

[28] G. Piro, L. Grieco, and G. Boggia, "Simulating LTE cellular systems: An open-source framework," IEEE Trans. Veh. Technol., vol. 60, no. 2, pp. 498 - 513, 2010. [Online]. Available: http://ieeexplore.ieee.org/xpls/abs $\backslash \_$all.jsp?arnumber=5634134

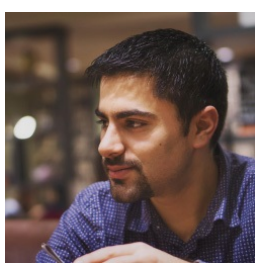

Dr. Louis Christodoulou received an MEng degree in Electronic Engineering with First Class Honours from the University of Surrey, United Kingdom in 2012. He continued to join the multimedia communication systems research lab (I-LAB) at the University of Surrey where he completed his PhD in 2016. With experience working in the television broadcast industry, his main research interests are centered around future multimedia content delivery, mobile communications and radio resource management.

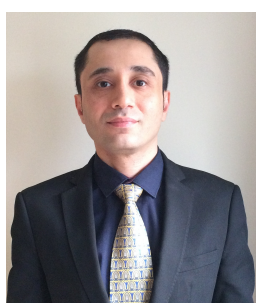

Dr. Omar Abdul-Hameed received his BEng degree in Electronic and Electrical Engineering with a First Class Honours and the Ph.D. degree from the University of Surrey, Guildford, UK in 2003 and 2009, respectively. From 2007 to 2014, he was a Research Fellow at the I-Lab, Multimedia Communications Research Centre at the University of Surrey, where he was actively involved and working in several European Commission funded FP6 and FP7 collaborative research projects. $\mathrm{He}$ then joined the Institute for Digital Technologies (IDT) at Loughborough University London in October 2014 as a Research Associate. His main research interests include technologies for $5 \mathrm{G}$ mobile communications, radio resource management in mobile broadband networks, the efficient and robust video content delivery over wireline and wireless networks, and experimental test-bed design and implementation with IP-based multimedia applications. He has several publications in international journals and conferences as well as several book chapters.

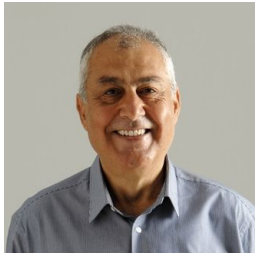

Professor Ahmet Kondoz received his $\mathrm{PhD}$ in 1987 from the University of Surrey where he was a research fellow in the communication systems research group until 1988. He became a lecturer in 1988, reader in 1995, and in 1996 he was promoted to professor in multimedia communication systems. He was the founding head of I-LAB, a multi-disciplinary multimedia communication systems research lab at the University of Surrey. Since January 2014, Prof. Kondoz has been appointed as the founding Director of the Institute for Digital Technologies, at Loughborough University London, a post graduate teaching, research and enterprise institute. He is also Associate Dean for Research for Loughborough University London. His research interests are in the areas of digital signal processing and coding, fixed and mobile multimedia communication systems, 3D immersive media applications for the future Internet systems, smart systems such as autonomous vehicles and assistive technologies, big data analytics and visualisation and related cyber security systems. He has over 400 publications, including six books, several book chapters, and seven international patents, and graduated more than $75 \mathrm{PhD}$ students. He has been a consultant for major wireless media industries and has been acting as an advisor for various international governmental departments, research councils and patent attorneys. He is a director of MulSys Ltd., a university spinoff company marketing the worlds first secure GSM communication system through the mobile voice channel. Professor Kondoz has been involved with several European Commission FP6 \& FP7 research and development projects, such as NEWCOM, e-SENSE, SUIT, VISNET, MUSCADE etc. involving leading universities, research institutes and industrial organisations across Europe. He coordinated FP6 VISNET II NoE, FP7 DIOMEDES STREP and ROMEO IP projects, involving many leading organisations across Europe which deals with the hybrid delivery of high quality $3 \mathrm{D}$ immersive media to remote collaborating users including those with mobile terminals. He cochaired the European networked media advisory task force, and contributed to the Future Media and 3D Internet activities to support the European Commission in the FP7 programmes.

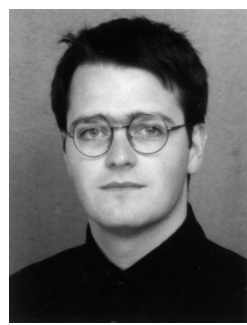

Dr Janko Calic is a Visiting Lecturer in Multimedia Systems and HCI at the I-Lab, a part of the Centre for Vision, Speech and Signal Processing at the University of Surrey. Previously, he was with the Department of Computer Science at University of Bristol in the period 2003-2007; and was awarded $\mathrm{PhD}$ and was the founding member of the Multimedia and Vision Research Group, at the Queen Mary, University of London between 2000-2003. His research focuses on multimedia communications and signal processing and human-computer interaction. Dr Calic regularly reviews for the leading international journals, conferences and book publishers ACM, IEEE, IEE, Elsevier, Springer, Wiley, and he has chaired special sessions and managed peer reviews at international conferences. 